\title{
Diet, physical activity and screen time but not body mass index are associated with the gut microbiome of a diverse cohort of college students living in university housing: a cross-sectional study
}

\author{
Corrie M. Whisner ${ }^{1,2^{*}}$ (D) Juan Maldonado ${ }^{2,3,4}$, Brandon Dente ${ }^{1}$, Rosa Krajmalnik-Brown ${ }^{2,4,5}$ and Meg Bruening ${ }^{1}$
}

\begin{abstract}
Background: Modifiable lifestyle factors (e.g. dietary intake and physical activity) are important contributors to weight gain during college. The purpose of this study was to evaluate whether associations exist between body mass index, physical activity, screen time, dietary consumption (fat, protein, carbohydrates, and fiber), and gut microbial diversity during the first year of college. Racially/ethnically diverse college students ( $n=82 ; 61.0 \%$ non-white) at a large Southwestern university completed self-reported physical activity and 24-h recall dietary assessments, height and weight measurements, and provided one fecal sample for gut microbiome analysis. Fecal microbial community composition was assessed with Illumina MiSeq next-generation sequencing of PCR amplified 16S rRNA genes. Post-hoc analyses compared microbial diversity by groups of high and low physical activity and fiber intake using QIIME and LEfSe bioinformatics software.
\end{abstract}

Results: No statistically significant differences were observed between body mass index and gut microbiome abundance and diversity. Median daily consumption of dietary fiber was $11.2(7.6,14.9) \mathrm{g} / \mathrm{d}$, while the median self-reported moderate-to-vigorous physical activity (MVPA) was $55.7(27.9,79.3) \mathrm{min} / \mathrm{d}$ and screen time 195.0 (195.0, 315.0) $\mathrm{min} / \mathrm{d}$. Microbial analysis by LEfSe identified Paraprevotellaceae, Lachnospiraceae, and Lachnospira as important phylotypes in college students reporting greater MVPA, while Enterobacteriaceae and Enterobacteriales were more enriched among students reporting less MVPA $(p<0.05)$. Barnesiellaceae, Alphaproteobacteria, and Ruminococcus were more abundant taxa among those consuming less than the median fiber intake $(p<0.05)$. Posthoc analyses comparing weighted UniFrac distance metrics based on combined categories of high and low MVPA and fiber revealed that clustering distances between members of the high MVPA-low fiber group were significantly smaller when compared to distances between members of all other MVPA-fiber groups $(p<0.0001)$.

Conclusions: Habitual fiber consumption and MVPA behaviors help explain the differential abundance of specific microbial taxa and overall gut microbial diversity differences in first-year college students.

Keywords: Microbiome, Microbiota, Adolescence, Pediatric, Obesity, Physical activity, Diet, Lifestyle behaviors

\footnotetext{
* Correspondence: cwhisner@asu.edu

${ }^{1}$ College of Health Solutions, Arizona State University, Phoenix, AZ, USA

${ }^{2}$ Center for Fundamental and Applied Microbiomics, Biodesign Institute,

Arizona State University, Tempe, AZ, USA

Full list of author information is available at the end of the article
}

(c) The Author(s). 2018 Open Access This article is distributed under the terms of the Creative Commons Attribution 4.0 International License (http://creativecommons.org/licenses/by/4.0/), which permits unrestricted use, distribution, and reproduction in any medium, provided you give appropriate credit to the original author(s) and the source, provide a link to the Creative Commons license, and indicate if changes were made. The Creative Commons Public Domain Dedication waiver (http://creativecommons.org/publicdomain/zero/1.0/) applies to the data made available in this article, unless otherwise stated. 


\section{Background}

Obesity remains a persistent public health issue affecting 78.6 million adults in the United States [1]. The transition from high school to college known as emergent adulthood is a vulnerable period of life which is frequently characterized by weight gain [2-6], making it an important period for prevention and intervention [5, 7]. This transition period is usually marked by leaving home for the first time, a new environment, building new friendships and social networks, and greater independence in overall decision making [2]. The incidence of obesity during this transition period is reportedly high and is thought to persist throughout adulthood [8,9]. Obesity is influenced by various factors including the environment [10], dietary intake [11], physical activity $[12]$, and the intestinal microbiome $[13,14]$. Higher incidence of obesity increases the risk of cardiometabolic conditions including hypertension, dyslipidemia, type 2 diabetes mellitus, heart disease, and cancer [9, 15].

Recent research suggests possible links between modifiable lifestyle factors, the gut microbiome, and health outcomes including obesity $[16,17]$. Findings for physical activity among college students are bleak, with most studies suggesting a lack of physical activity among this population [6, 18-20]. First-year students specifically, have reported that the transition to college makes it difficult to maintain health and physical activity due to lack of intrinsic motivation, loss of routine, and fewer opportunities for organized sports participation [21]. Decreases in physical activity have been associated with changes in body composition, including increased fat mass and decreased lean body mass [22]. This suggests that changes in body composition may be a sensitive indicator of lifestyle changes during the freshman year.

Dietary behaviors adopted by college students frequently include meals at 'all-you-can-eat' facilities, evening snacking, junk food consumption, and dieting; these behaviors have all been associated with weight gain [23]. Changes in dietary behaviors ultimately result in greater consumption of energy-dense, nutrient-poor foods such as sugar-sweetened beverages, fried foods, and salty snacks $[6,24]$. Ultimately these foods displace nutrientrich fruits, vegetables and whole grains containing dietary fiber which promote the development of a healthy gut microbiome. College students habitually have inadequate $(\sim 18 \mathrm{~g})$ dietary fiber consumption [25].

Studies show that dietary alterations can rapidly modify the gut microbiome [26]. Switching mice from a low-fat, plant-based diet to a high-fat/high-sugar diet negatively impacted the gut microbiome composition $[26,27]$. A comparison of children from Western Europe and Burkina Faso (BF) revealed significant differences in the gut microbiome composition with high dietary fiber consumption among BF children resulting in greater
Bacteroidetes relative to Firmicutes [28]. While components of plant-based diets have been reviewed and are thought to increase gut microbiome diversity via their dietary fiber composition [29], the influence of physical activity on the gut microbiome is an emerging area of research with very few human studies, as recently reviewed [30].

Gut microbiota composition differences have been observed between active and sedentary women with health-associated microbes Akkermansia muciniphila, Fecalibacterium prausnitzii, Bifidobacteria longum, and Roseburia hominis being more abundant in women meeting daily physical activity recommendations [31]. Faecalibacterium and Roseburia were also abundant in type-1 diabetics and healthy controls matched for high physical fitness with no differences by health status [32]. Specifically, aerobic exercise training for 6 weeks appears to shift the gut microbial composition and function as greater similarities were observed between obese and lean individuals irrespective of dietary intake [33]. Conversely, a study among elite rugby athletes found that both protein consumption and physical activity increased microbial diversity when compared to obese and normal weight controls [34]. One other study among women with breast cancer suggested that fitness level did not significantly associate with the relative abundance of specific gut microbes [35].

The gut during childhood and adolescence exhibits greater interpersonal variation and lower bacterial diversity compared to adults [36, 37]. This reduced diversity appears to create a more plastic and malleable gut microbiome [36, 37] which may fuel growth and allow greater and lasting microbial shifts in response to physical activity and diet. University students living on campus generally experience major changes in lifestyle habits, including physical activity and diet [29, 38]. Given that behaviors established during the college years may persist throughout adulthood and increase the risk of obesity and cardiometabolic diseases, this population provides a unique opportunity to expand our understanding of the role of physical activity and diet on the gut microbiome. The objective of this cross-sectional, observational study was to characterize the gut microbiome of a racially/ethnically diverse cohort of college students living in the dorms and assess possible associations with body mass index, measures of physical activity (moderate-to-vigorous physical activity), sedentary behavior (screen time), and dietary (fat, protein, carbohydrates, and fiber) intake.

\section{Results}

Participant characteristics

A total of 82 participants (57.3\% female; $31.7 \%$ Hispanic) provided a fecal sample, MVPA and screen time data 
(Table 1). A subsample $(n=68 ; 60.3 \%$ female; $57.3 \%$ non-white) also provided $24-\mathrm{h}$ dietary recall data. The median (IQR) percentage of kilocalories consumed from protein, fat and carbohydrate were $16.2(14.2,18.8) \%$, $35.8(30.0,40.8) \%$, and $47.9(39.7,54.4) \%$, respectively (Table 1). Both protein and carbohydrate consumption were within the acceptable macronutrient distribution range (AMDR) of $10-35 \%$ and $45-65 \%$, respectively; while the median fat consumption fell slightly outside the AMDR range of $20-35 \%$ [39]. The median (IQR) self-reported daily intake of sugar consumed was 65.5 $(47.6,104.6) \mathrm{g} / \mathrm{d}$. Median daily consumption of dietary fiber for males $(n=27)$ and females $(n=41)$ was 8.7 $(7.1,14.2) \mathrm{g} / \mathrm{d}$ and $11.4(8.6,16.8) \mathrm{g} / \mathrm{d}$, respectively, for which both fell below the AMDR for males $(38 \mathrm{~g} / \mathrm{d}$ ) and females (25-26g/d) [39]. Self-reported MVPA $(p=0.133)$ and screen time $(p=0.441)$ did not differ by BMI classification. Self-reported screen time and MVPA were not significantly correlated (Spearman rho $=-0.143, p=0.199$ ).

Table 1 Sociodemographic and key variables of college students living in residence halls $(n=82)$

\begin{tabular}{|c|c|}
\hline Variable & \\
\hline Age (years) mean $\pm S D$ & $18.4 \pm 0.6$ \\
\hline \multicolumn{2}{|l|}{$\operatorname{Sex} \%(n)$} \\
\hline Male & $42.7(35)$ \\
\hline Female & $57.3(47)$ \\
\hline \multicolumn{2}{|l|}{ Residence hall \% (n) } \\
\hline A & $37.8(31)$ \\
\hline B & $62.2(51)$ \\
\hline \multicolumn{2}{|l|}{ Race/ethnicity \% (n) } \\
\hline Hispanic & $31.7(26)$ \\
\hline White & $39.0(32)$ \\
\hline Other & $29.3(24)$ \\
\hline Body Mass Index $\left(\mathrm{kg} / \mathrm{m}^{2}\right)$ mean $\pm S D$ & $24.4 \pm 5.5$ \\
\hline$<18.5 \mathrm{~kg} / \mathrm{m}^{2} \%(n)$ & $6.1(5)$ \\
\hline $18.5-24.9 \mathrm{~kg} / \mathrm{m}^{2} \%(n)$ & $57.3(47)$ \\
\hline $25.0-29.9 \mathrm{~kg} / \mathrm{m}^{2} \%(n)$ & $22.0(18)$ \\
\hline$\geq 30.0 \mathrm{~kg} / \mathrm{m}^{2} \%(n)$ & $14.6(12)$ \\
\hline \multicolumn{2}{|l|}{ Diet $^{\mathrm{a}}$ median (IQR) } \\
\hline Carbohydrates (g) & $165.7(125.1,240.7)$ \\
\hline Fiber (g) & $11.2(7.6,14.9)$ \\
\hline Protein (g) & $61.8(42.4,85.4)$ \\
\hline Fat (g) & $63.3(38.8,84.8)$ \\
\hline $\begin{array}{l}\text { Moderate-to-vigorous physical activity (min/day) } \\
\text { median (IQR) }\end{array}$ & $55.7(27.9,79.3)$ \\
\hline Screen time (min/day) median (IQR) & $195.0(195.0,315.0)$ \\
\hline
\end{tabular}

a Sample size decreases to $n=68$ for diet data due to missing 24-h dietary recalls; $I Q R$, interquartile range; $S D$, standard deviation

\section{Gut microbiota and behaviors}

Amplicon high-throughput sequencing resulted in an average of $60,00016 \mathrm{~S}$ rRNA gene amplicon reads per sample. Rarefaction curves based on observed species, Chao1 and Faith's PD (phylogenetic diversity) metrics suggested that adequate sampling depth was at 17,768 sequences. The median Firmicutes:Bacteroidetes ratio was $0.65(0.39,1.23)$. This ratio did not differ by BMI group ( $p=0.413$ ) or median categories of dietary protein $(p=0.763)$, fat $(p=0.469)$, carbohydrate $(p=0.683)$, and fiber $(p=0.835)$ intake. Similarly, the F:B ratios between MVPA $(p=0.583)$ and screen time $(p=0.323)$ categories did not differ significantly.

Chao1, observed OTU, and PD whole tree alphadiversity did not differ significantly by categories (above or below the median) of dietary fat, protein, carbohydrate, or fiber. While no obvious visual differences in beta diversity (between-sample) were observed via PCoA plots (Additional file 1), comparison of distributions of distances showed significant within-group differences for carbohydrate, fiber and protein using both unweighted and weighted UniFrac data (weighted data are shown in Fig. 1). For fat consumption, only the within-group weighted UniFrac distances were significantly different between high and low-fat. Despite these differences in beta-dispersion, PERMANOVA results indicated that between group differences in weighted and unweighted distance metrics were not significantly influenced by dietary factors.

Chao1, observed OTUs, and PD whole tree alphadiversity metrics did not differ significantly by categories of MVPA or screen time, suggesting that physical activity and sedentary behaviors were not associated with species richness or evenness in this cohort of college students. No obvious visual differences in beta diversity (between-sample) were observed via PCoA plots (Additional file 1) when evaluating MVPA and screen time quartiles. Upon comparison of UniFrac distance metrics with Bonferroni corrections, significant differences in beta diversity were observed between MVPA groups when evaluating the median unweighted but not weighted within-group UniFrac distances (Fig. 2a). As unweighted UniFrac distances help to explain the presence of less abundant, rather than most abundant taxa; this result suggests that less abundant taxa may differ by self-reported physical activity levels. Both unweighted and weighted UniFrac within-group distances differed by self-reported total daily sedentary time or time spent in front of a screen (Fig. 2b and c). These data suggest that presence of both less and most abundant taxa may be characteristic of students with less $(2.5-4 \mathrm{~h})$ sedentary time compared to all other groups. Despite within-group differences in distances, no significant between-group differences in weighted and 


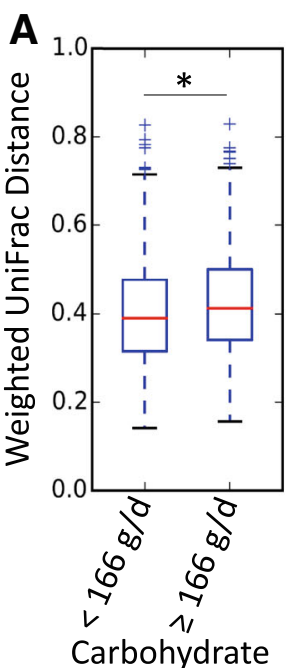

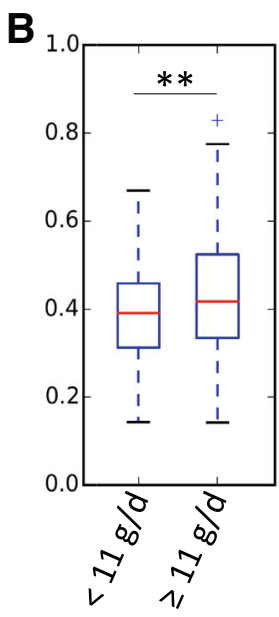

Fiber

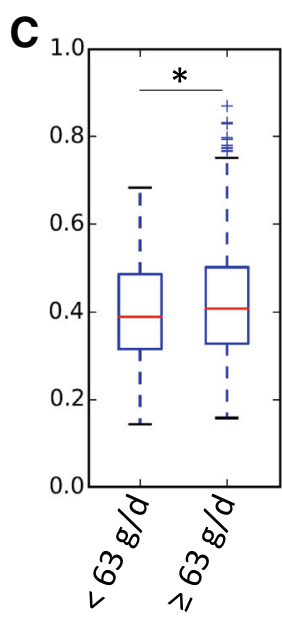

Fat

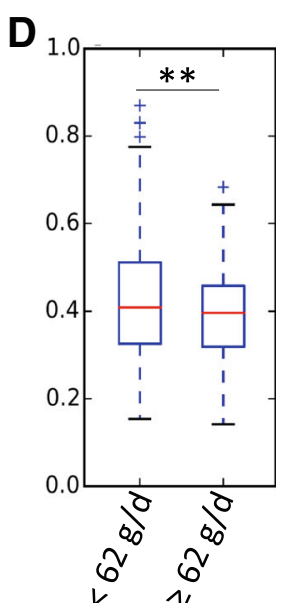

Protein

Fig. 1 Distance metrics by (a) carbohydrate, (b) fiber, (c) fat, and (d) protein consumption groups. Groups were created by separating self-reported values that fell above and below median daily intakes. Dietary intake was obtained from automated self-report $24-\mathrm{h}$ dietary recalls. Significant differences in distance metrics between members of one group compared to another are denoted as * $p<0.05$, ** $p<0.001$

unweighted distances were observed for MVPA and sedentary time, as analyzed by PERMANOVA.

To further explore the influence of MVPA and dietary fiber consumption, in post-hoc analyses, we examined the beta-dispersion (weighted and unweighted UniFrac distances) within groups of participants characterized by merged MVPA and fiber categories (Group 1: low MVPA-low fiber, Group 2: low MVPA-high fiber, Group 3: high MVPA-low fiber, and Group 4: high MVPA-high fiber). Unweighted UniFrac data suggested that the high MVPA-low fiber group (Group 3) had significantly shorter distances among group members when compared to within-group distances of all other MVPAfiber combination groups (Fig. 3, $p<0.001$ ). When distances were partitioned by group and compared via PERMANOVA, no significant between-group differences were observed.

Analyses of fecal microbiota in high and low (median cut point) MVPA or dietary fiber groups using LEfSe allowed for the identification of specific bacterial taxa that were associated with self-reported MVPA and dietary fiber consumption. The greatest differences at various taxa levels between the two communities are displayed for MVPA (Fig. 4a) and dietary fiber (Fig. 4b).
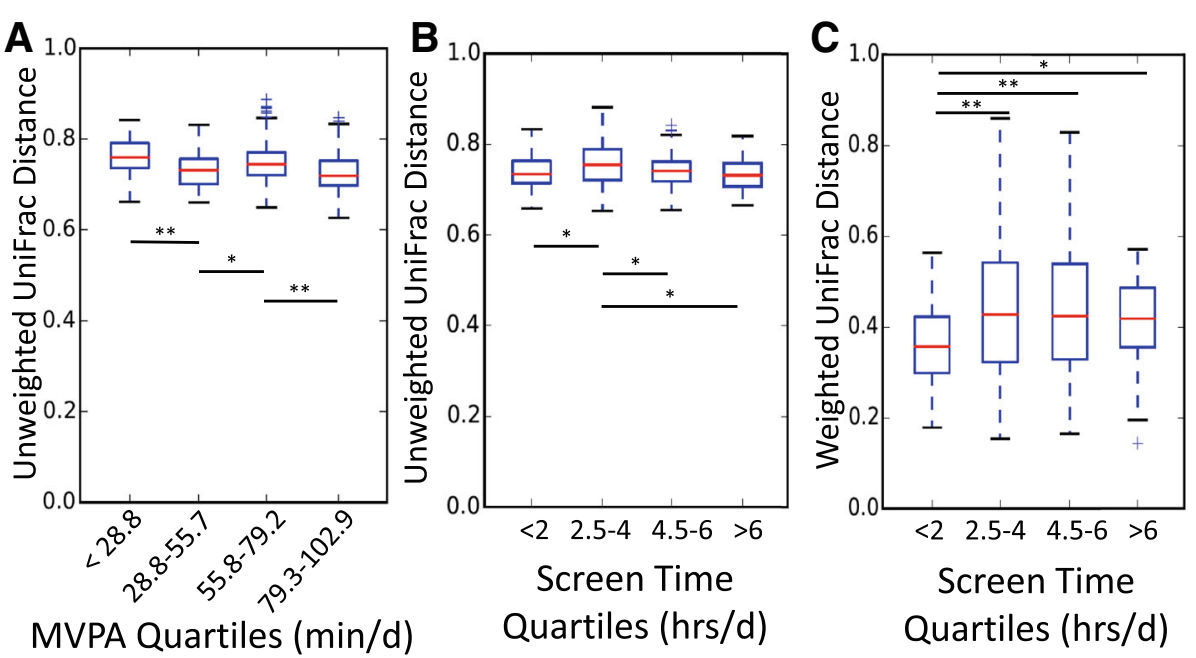

Fig. 2 Differences in distance metrics by self-reported (a) MVPA and (b and c) screen time. MVPA and screentime were self-reported using validated survey questions. Significant differences in distance metrics between members of one group compared to another group are denoted as ${ }^{*} p<0.05 ;{ }^{* *} p<0.0001$. MVPA, moderate-to-vigorous physical activity 


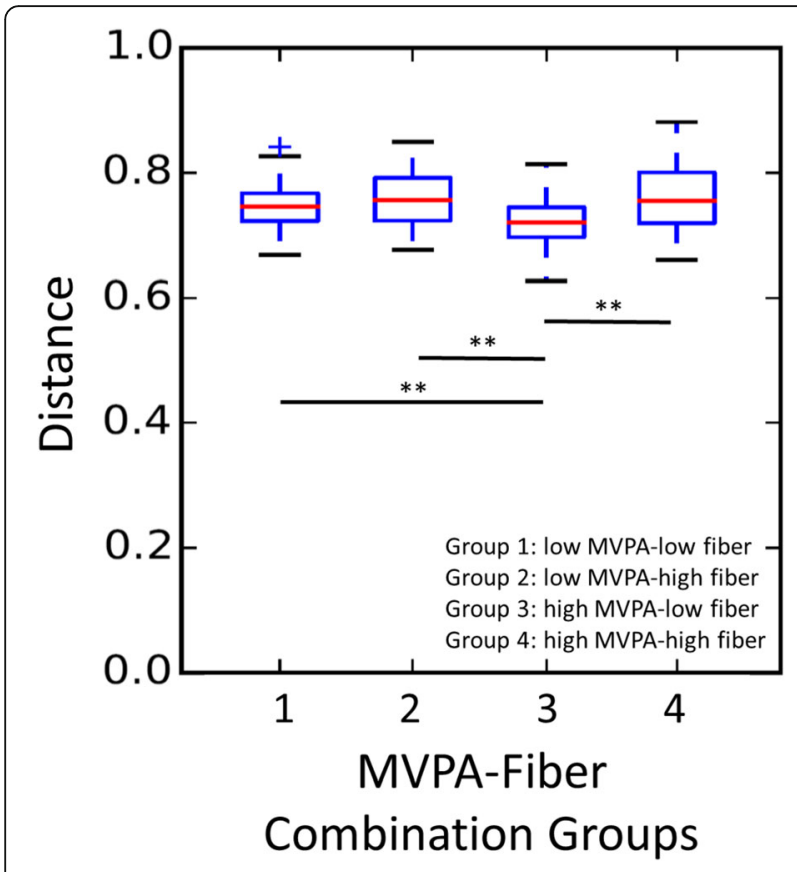

Fig. 3 Beta diversity (unweighted UniFrac data) by categories of combined high and low MVPA and fiber. Median MVPA (55.7 min/d) and dietary fiber $(11 \mathrm{~g} / \mathrm{d}$ ) consumption were used to create high and low groupings. Significant differences in distance metrics between members of one group compared to another group are denoted as ${ }^{* *} p<0.0001$. MVPA, moderate-to-vigorous physical activity

Data for MVPA suggested significant enrichment of family Paraprevotellaceae among those reporting greater MVPA. Family Lachnospiraceae and its genus Lachnospira were also identified as potential microbial markers of this more active group of college students. Family Enterobacteriaceae and genus member Enterobacteriales were more enriched among college students reporting MVPA below the median value of $55.7 \mathrm{~min} / \mathrm{d}$. The family Barnesiellaceae, class Alphaproteobacteria, and genera Ruminococcus and unassigned Bacteroidales were more abundant taxa in the low fiber consumption group, while Tenericutes and other unassigned microbes were more abundant among those consuming greater than the median dietary fiber intake.

\section{Discussion}

This study is unique given its focus on college students, a population susceptible to major lifestyle changes that occur during a period of continued social and physical development. In this study, we observed within-group differences in beta-dispersion among a diverse cohort of first-year college students reporting different dietary and physical activity behaviors but these behavioral categories did not explain between-group differences in microbial community structure. Additionally, a merged comparison of dietary fiber consumption and MVPA revealed differences in microbial beta-dispersion such that high MVPA combined with low fiber intake resulted in smaller within-group distances when compared to within-group distances for all other MVPA-fiber groups. Lastly, we report that specific microbial taxa were differentially abundant among college students reporting different daily MVPA and fiber consumption habits.

This study revealed no difference in the F:B ratio by BMI, dietary intake variables, MVPA or screen time categories. Previous literature has highlighted contradictory results with regard to this phyla-level assessment. Firmicutes have frequently been shown to decrease with weight loss and have been observed in higher proportions among obese animals and humans when compared to lean counterparts [40-42]. Nonetheless, others have reported no difference in the F:B ratio in relation to BMI or weight change [43-45] or a greater abundance of Bacteroidetes among individuals with increased BMIs [46]. Bacteroidetes have been positively associated with dietary fat while Firmicutes have been associated with dietary fiber [47]. Conversely, a study in children reported greater Bacteroidetes among those consuming low-fat, high-starch and fiber diets when compared to children consuming more western-style diets [28]. Still other studies report that diet did not alter the proportions of Firmicutes and Bacteroidetes [48]. Although such discrepancies also exist among the emerging literature regarding physical activity, exercise and the gut microbiome, these differences in findings may be the result of differing study designs, methodologies, sample types, and lack of replication of data. Similar to our findings, the F:B ratio did not differ among exercise-trained, obesity-prone rats [49] or active adult women [31], when compared to sedentary controls. To date, three studies have suggested a decrease in Firmicutes among exercisetrained rodents of varying metabolic status [50-53], while two investigations suggested an increase in Firmicutes among exercise-trained rodents [54-56].

Findings from the current study suggest that gut microbial beta-dispersion but not between-group distances differed by categories of high and low macronutrient (carbohydrate, protein and fat) consumption. Carbohydrates, in particular dietary fibers, have been identified as an important fuel source for the gut microbiome as part of habitual diets and short- and long-term dietary interventions [57]. The finding that decreased dietary fiber consumption among college students resulted in beta-dispersion differences may be supported by data suggesting that dietary fiber increases microbial diversity in the gut by altering the composition of fiber-fermenting microbes [28, 58]; however, causality cannot be inferred in this cross-sectional analysis. Previous assessments of lifestyle factors and gut microbiome community structure also support our findings of 

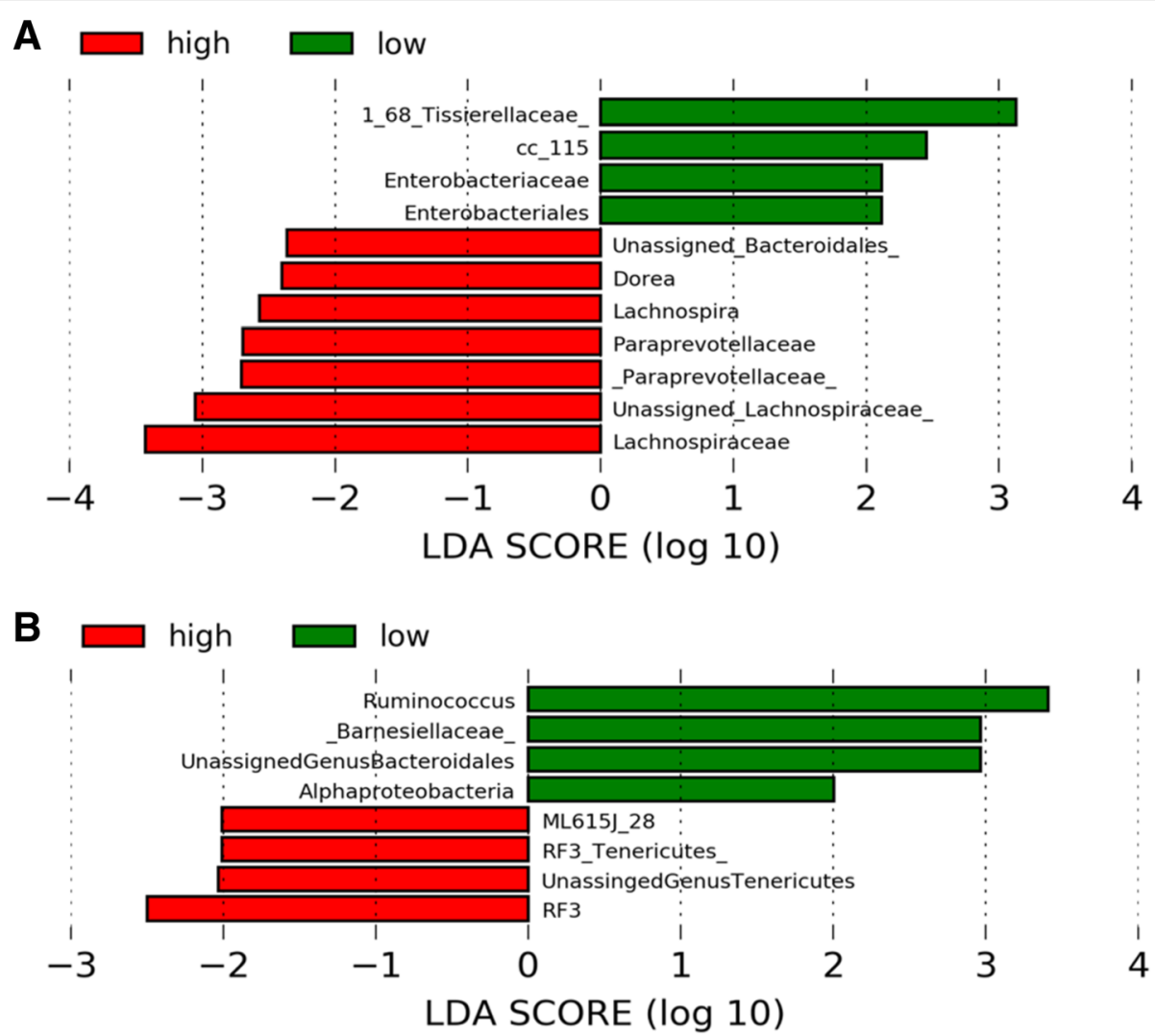

\section{4}
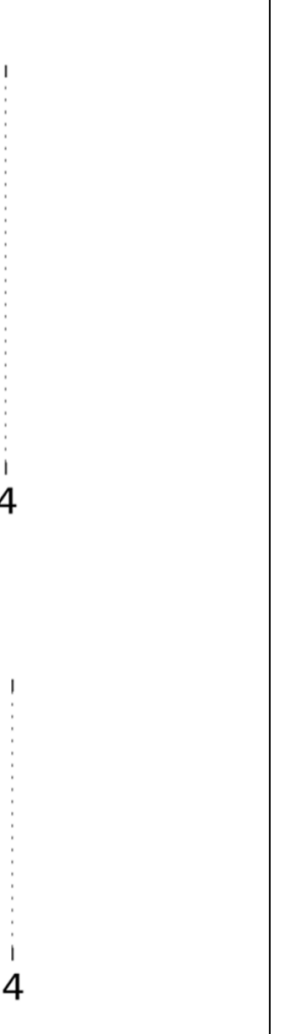

Fig. 4 Differential microbial abundance between high and low (a) MVPA and (b) dietary fiber intake groups. High and low MVPA and fiber groups were greater than or less than median self-reported physical activity $(55.7 \mathrm{~min} / \mathrm{d})$ and dietary fiber consumption $(11.2 \mathrm{~g} / \mathrm{d})$, respectively. Identification of differentially abundant microbial taxa was done by linear discriminant analysis effect size analyses (LEfSe). MVPA, moderate-to-vigorous physical activity

increased abundance of Barnesiellaceae, Alphaproteobacteria, and Ruminococcus, and decreased Tenericutes among low-fiber consuming students compared to high-fiber consumers. Barnesiellaceae members have been associated with Western diets limited in fiber [59]. Ruminococcus has been associated with the degradation of resistant starches which remain abundant in processed refined grain products [59]; this may explain the observed increase among low-fiber consumers. Tenericutes had a greater relative abundance among Bangladeshi children consuming fiber-rich diets compared to American children following Western diets higher in fat and protein and low in fiber [60]. Findings related to dietary fiber in the current study occurred despite the fact that college students in the present study reported consuming a median fiber intake slightly more than 11 $\mathrm{g} / \mathrm{d}$. While this falls well below recommended intakes for males and females $38 \mathrm{~g} / \mathrm{d}$ and $25-26 \mathrm{~g} / \mathrm{d}$, respectively [39], this finding suggests that small amounts of dietary fiber may be sufficient to model potentially positive changes in the gut microbiome. Further work is needed to assess specific types of fiber and their influence on specific microbial taxa and evaluate cause and effect relationships in both animals and humans.

Gut microbial taxa varied among students reporting differing levels of daily physical activity with Paraprevotellaceae, Lachnospiraceae, and Lachnospira being more prevalent in college students reporting greater MVPA, and Enterobacteriaceae and Enterobacteriales being more enriched among college students reporting less MVPA. To date, the majority of evidence for exercise and the gut microbiome is from animal models. A pilot study among elite cyclists also found that Prevotella genera abundance was positively correlated with the amount of time spent exercising [61]. Similar to our findings, although in a mouse model with longitudinal measures, Evans et al. reported an increase in Lachnospiraceae with voluntary wheel running and an increase in Ruminococcaeae but this family may have been equally influenced by the high-fat feeding protocol [50]. In a study of mice with ad-libitum food access, voluntary wheel running resulted in increased fecal Lactobacillus, Bifidobacterium and Blautia coccoides-Eubacterium rectale and decreased Clostridium and Enterococcus 
when compared to sedentary controls [53]. Voluntary wheel running among mice has also been shown to revert the negative effects of polychlorinated biphenyl exposure on the gut microbiome with significantly different community structures compared to sedentary mice [51]. Mode of exercise may also play a role in shaping the gut microbiome, as high-intensity interval training 3 times per week for 6 weeks improved the diversity of the colonic gut microbiota in high-fat fed mice [52]. While our study did not capture the types of physical activity in which students were engaging, a recent human study found that endurance exercise-induced changes in the gut microbiota were dependent on body mass suggesting that the metabolic health of individuals should be accounted for in future studies [33]. The majority of participants in the current study were of normal weight and metabolically healthy. We did not observe differences in gut microbiome diversity by BMI in our study (mean \pm SD: $24.4 \pm 5.5 \mathrm{~kg} / \mathrm{m}^{2}$, range: $16.9-50.4 \mathrm{~kg} / \mathrm{m}^{2}$ ).

While animal models have been helpful for establishing relationships between the gut microbiome, physical activity and exercise, these links have been more difficult in humans. To date, there have been few human studies. A recent study found that the gut microbiome of adult women meeting the World Health Organization recommendations for physical activity differed from that of sedentary women with significantly greater abundance of species associated with metabolic health [31]. Other studies have primarily focused on elite athletes who have a tendency toward extreme dietary and exercise behaviors which make it difficult to extrapolate results to the general public [34, 61]. A study conducted on elite rugby players suggested that the gut microbiome of athletes differed significantly in comparison to healthy weight and obese sedentary controls; however, these community differences also appeared to be influenced by the unique dietary practices of the athletes [34]. In our study, we aimed to assess potential dietary and physical activity interactions by comparing beta-diversity patterns between merged groups of high and low MVPA and fiber. We found that high MVPA combined with low fiber intake resulted in significantly different beta-dispersion than all other combinations of MVPA and fiber consumption but that between-sample distances did not differ by MVPA-fiber categories. This suggests that betadispersion patterns might be a marker of specific physical activity and dietary behaviors but inferring causality is not possible given that other unmeasured factors might also be impactful. Kang et al. have reported differential clustering due to exercise on both normal and high-fat diet-fed rats [54] while Welly et al. did not find orthogonal differences in PCoA plots between exercised and dietary-restricted obese rats [49]. Previous work has reported both increases [62] and decreases [51] in fecal Tenericutes phylum relative abundance among exercised animals, while our study and work by Lin et al. found that this taxa may differ by fiber-rich foods (legumes, grains) consumption [60]. Work by Kang et al. also suggests that exercise influences Tenericutes as this taxa increased despite consumption of standard or high-fat diets [54]. Differential effects on individual gut microbiome taxa require further exploration to better understanding how physical activity and diet independently and mutually influence health.

Limitations of the current study include the small sample size which make it difficult to assess demographic differences in dietary and MVPA behaviors. Further, conclusions regarding gut microbial data should be made with caution as this study was cross-sectional (cannot infer causality) and the collection of a single fecal sample may not accurately capture gut microbiome differences in a free-living population where environmental exposures, diet, physical activity, and other behaviors vary from day to day. Despite not being able to characterize the specific types of physical activity in which college students were engaging at the time of assessment, a strength of this study was the use of validated self-report instruments for assessment of MVPA and dietary intake. Studying a diverse cohort of college students is also a strength as the current microbiome literature has largely ignored this age group and infrequently includes individuals from all races and ethnicities.

\section{Conclusions}

In summary, this study provides observational support for the importance of regular physical activity in shaping the gut microbiome during a period of continued growth and development. Data from this study suggest that while beta-dispersion differed among high and low macronutrient consumers or physical activity categories, between-group distances were not significantly different among these categories. Specific taxa associated with health were differentially more abundant among those reporting greater self-reported fiber intakes and MVPA. While these results are promising, more research is warranted to fully elucidate the role of physical activity and diet in modulating the gut microbiome. Being one of the first studies to examine the gut microbiome in college-aged subjects, opportunities for further investigation include assessment of specific physical activities, exercise interventions assessing different modes and duration of activity, and evaluation of diet and physical activity interactions. Next steps will include hypothesis testing in suitable animal models and human cohorts that utilize carefully designed, longitudinal approaches to elucidate cause and effect relationships between 
dietary and physical activity effects on the gut microbiome. This work will further identify microbial biomarkers of health and enhance our understanding of how changes in diet and physical activity impact health outcomes including weight gain, a common health outcome among college students and humans of all ages.

\section{Materials and methods}

Healthy college students living in on-campus housing, who were English speaking, and at least 18 years of age were eligible to participate in this cross-sectional study. This cohort of eligible students were recruited from a larger study [63] that used mobile ecological momentary assessment methodology to assess the influence that social networks have on physical activity, dietary intake, and body weight in two residence halls at Arizona State University in Tempe, Arizona. Exclusion criteria for this study included a history of malabsorptive disorders, high blood pressure, eating disorders, HIV infection, diabetes, and/or the use of antibiotics, antifungals, or probiotics in the 2 to 3 months prior to the study. This study was conducted during the Fall 2014 and Spring 2015 semesters. The Arizona State University (ASU) Institutional Review Board approved (STUDY00002019) this study and all participants provided written informed consent.

Participant age, gender, race/ethnicity, and other demographic data were provided via a self-reported, web-based questionnaire that was completed upon entry into the parent study. Height and weight measurements were measured by trained research staff. Each measurement was taken up to three times and the two closest values within $0.5 \mathrm{~cm}$ and $0.5 \mathrm{~kg}$ of each other, respectively, were averaged. These averaged values were then used to calculate body mass index (BMI) and categorize participants based on the CDC guidelines as follows: $\mathrm{BMI}<18.5 \mathrm{~kg} / \mathrm{m}^{2}$ was considered underweight; $\mathrm{BMI} \geq$ $18.5 \mathrm{~kg} / \mathrm{m}^{2}$ and $\leq 24.9 \mathrm{~kg} / \mathrm{m}^{2}$ was considered normal weight; $\mathrm{BMI} \geq 25.0 \mathrm{~kg} / \mathrm{m}^{2}$ and $\leq 29.9 \mathrm{~kg} / \mathrm{m}^{2}$ was considered overweight; and $\mathrm{BMI} \geq 30.0 \mathrm{~kg} / \mathrm{m}^{2}$ was considered obese [64].

Physical activity habits were determined using the Godin-Shephard Leisure-Time Physical Activity Questionnaire (see Additional file 2) [65]. The Godin-Shephard protocol has been validated as an appropriate method to measure physical activity habits in college-aged males and females [65]. Sedentary activities were also measured using a validated survey (Additional file 2) [66].

The ASA24 24-h dietary recall was used to assess students' habitual dietary intake. Food and beverage intake was recorded from midnight to midnight on the previous day. The website provided images to guide participants on selecting the correct portion size for each item they consumed. Participants were asked to complete 3 days of dietary recall ( 2 weekdays and 1 weekend day) which has been validated as a representative and accurate summary of habitual nutrient intake $[67,68]$. Days of intake were dropped if caloric intake was below 500 or in excess of $5000 \mathrm{kcal}$. If a participant did not have at least 1 day of adequate dietary intake they were excluded from the study. The validated ASA24 [69] utilizes the US Department of Agriculture's Automated Multiple Pass Method (AMPM) [70] and measures intake by using the USDA's Food and Nutrient Database for Dietary Studies (FNDDS). Using data from the ASA24-2014 Daily Total Nutrients Analysis File (TN), we examined total grams of protein, fat, carbohydrates, and fiber.

Each study participant was provided with a fecal sample collection kit (Commode Specimen Collection Kit, Fisher Scientific, Anthem, AZ) in order to provide a single fecal sample for analysis. Collection kits were distributed to participants in small insulated cooler bags containing ice packs to keep samples cold while in transit post-collection. Before participants left with the kit, a brief demonstration on how to collect the sample was provided along with a sheet of instructions inside the cooler bag. Participants were asked to freeze their ice packs immediately so that they were frozen at the time of sample collection. Ice packs were rated to stay frozen for $36-48 \mathrm{~h}$ in an insulated container. All stool samples were retrieved from participants and delivered to the clinical research facility within $24 \mathrm{~h}$ of collection. Stool samples were stored at $-80^{\circ} \mathrm{C}$ to preserve the microbial community.

Assessment of the gut microbiome in fecal collections was carried out at the Biodesign Institute at ASU in Tempe, Arizona. Extraction of microbial DNA from fecal samples was accomplished using the PowerSoil DNA isolation kit as described by the manufacturer (MoBio Laboratories Ltd., Carlsbad, CA) using a beadbeater (BioSpec, Bartlesville, OK). Amplification of the $16 \mathrm{~S}$ rRNA gene sequence was completed in triplicate PCRs using 96-well plates. Barcoded universal forward 515F primers and 806 R reverse primers containing Illumina adapter sequences, which target the highly conserved V4 region, were used to amplify microbial DNA $[71,72]$. These primers were selected as they are recommended by the Earth Microbiome Project [71, 72] and the National Institutes of Health Human Microbiome Project [73] to enhance reproducibility and comparability to other studies while obtaining broad coverage of Bacteria. PCR, amplicon cleaning and quantification were performed as previously outlined [72]. Equimolar ratios of amplicons from individual samples were pooled together before sequencing on the Illumina platform (Illumina MiSeq instrument, Illumina, Inc., San Diego, CA) at ASU's DNASU Genomics Core Facility. Raw 
Illumina microbial data were cleaned by removing short and long sequences, sequences with primer mismatches, uncorrectable barcodes, and ambiguous bases using the Quantitative Insights Into Microbial Ecology (QIIME) software, version 1.9.1, as previously described [74]. Taxonomic assignments and operational taxonomic units (OTUs) were determined using the closed reference Greengenes database [75] at 99\% similarity. The OTU table was filtered for singletons by using the QIIME script filter_otus_from_otu_table.py. OTUs observed fewer than two times were removed from the table.

All statistical analyses were completed using JMP Pro 13 and QIIME 1.9.1 statistical and bioinformatics software packages. Data were expressed as mean $\pm S D$ or median (interquartile range) of microbiota frequencies/ proportions based on the normality of the data. BMI data were expressed both continuously and categorically (underweight, normal weight, overweight, and obese). Wilcoxon-Kruskal Wallis tests were carried out to assess group differences (e.g. BMI, screen time, MVPA) in the Firmicutes:Bacteroidetes (F:B) ratio. Phylogenetic diversity measures were carried out in QIIME to determine alpha (within-sample) diversity metrics via Faith's PD. Principal coordinates analysis (PCoA) was performed for beta (between-sample) diversity analysis, using both weighted and unweighted unique fraction metric (UniFrac) distances (measure of phylogenetic distance between sets of taxa in a phylogenetic tree as a fraction of the branch length on the tree), on the 99\% OTU composition and abundance matrix [76]. UniFrac distance metrics group comparisons were performed for self-reported MVPA, screen time, and dietary intake variables. Linear discriminant analysis (LDA) effect size (LEfSe) was performed to identify microbial taxa that were differentially abundant by MVPA and dietary fiber consumption groups [77]. Findings were considered significant at $p<0.05$ following adjustments for multiple comparisons. Sample sequences were deposited at the NCBI/Sequence Read Archive (SRA) under project PRJNA473006 with accession numbers: SAMN09258197 - SAMN09258278.

\section{Additional files}

Additional file 1: File showing PCoA plots for all dietary intake, physical activity, and screen time group median or quartile comparisons. (PDF $1045 \mathrm{~kb}$ )

Additional file 2: Physical Activity and Sedentary Activity Questionnaire Details. This file offers additional information regarding questions used in survey instruments and scoring methods to tabulate or summarize data for statistical analyses. (PDF $69 \mathrm{~kb}$ )

Additional file 3: Table showing participant characteristics including demographics, behavioral data and alpha diversity metrics. (XLSX $21 \mathrm{~kb}$ )

Additional file 4: Table showing taxonomic abundance data for all genera. (XLSX $113 \mathrm{~kb})$

\section{Abbreviations}

AMPM: US Department of Agriculture's Automated Multiple Pass Method; BMI: Body mass index; F:B: Firmicutes:Bacteroidetes; FNDDS: USDA's Food and Nutrient Database for Dietary Studies; LDA: Linear discriminant analysis; LEfSe: Linear discriminant analysis (LDA) effect size; MVPA: Moderate-tovigorous physical activity; OTU: Operational taxonomic unit; PCOA: Principal coordinates analysis; UniFrac: Unique fraction metric

\section{Acknowledgements}

We would like to thank the students for taking the time to participate in this study. We would also like to thank the devilSPARC research team for their assistance in collecting the data.

\section{Funding}

This study was supported by the $\mathrm{NIH}$ Common Fund from the Office of the Director and the Office of Behavioral and Social Sciences Research, grant number 1DP5OD017910 (PI: M. Bruening). RKB is supported by the National Institute of Diabetes and Digestive and Kidney Diseases of the National Institutes of Health under Award Numbers R01DK090379 and R01DK105829. The content of this manuscript is solely the responsibility of the authors and does not necessarily represent the official views of the National Institutes of Health. All funding bodies had no role in the design of the study, nor the collection, analysis, and interpretation of data during manuscript preparation.

\section{Availability of data and materials}

The microbial sequence dataset supporting the conclusions of this article is available in the open-source repository "NCBI/Sequence Read Archive (SRA)" under project PRJNA473006 with accession numbers: SAMN09258197 -

SAMN09258278 (https://www.ncbi.nlm.nih.gov/sra). Relevant phenotype (behavioral and demographic variables) data are included within the article as Additional files 3 and 4 .

\section{Authors' contributions}

$M B$ wrote the funding proposal for the parent study. MB, CMW and RKB designed the study. MB, CMW and BD coordinated the study and gathered all study data. JM processed the biosamples and performed the data acquisition. JM and CMW performed the analysis and interpreted the data. CMW and BD wrote the main paper, and JM, RKB, and MB revised the manuscript. All authors read and approved the final manuscript.

\section{Ethics approval and consent to participate}

The Arizona State University (ASU) Institutional Review Board approved (STUDY00002019) this study and all participants provided written informed consent.

\section{Consent for publication}

Not applicable.

\section{Competing interests}

The authors declare that they have no competing interests.

\section{Publisher's Note}

Springer Nature remains neutral with regard to jurisdictional claims in published maps and institutional affiliations.

\section{Author details}

${ }^{1}$ College of Health Solutions, Arizona State University, Phoenix, AZ, USA. ${ }^{2}$ Center for Fundamental and Applied Microbiomics, Biodesign Institute, Arizona State University, Tempe, AZ, USA. ${ }^{3}$ ASU Genomics Core, Biodesign Institute, Arizona State University, Tempe, AZ, USA. ${ }^{4}$ Swette Center for Environmental Biotechnology, Biodesign Institute, Arizona State University, Tempe, AZ, USA. ${ }^{5}$ School of Sustainable Engineering and the Built Environment, Fulton Schools of Engineering, Arizona State University, Tempe, $A Z$, USA.

Received: 19 July 2018 Accepted: 2 December 2018

Published online: 12 December 2018

References

1. Centers for Disease Control and Prevention. Adult Obesity Causes \& Consequences. 2018. https://www.cdc.gov/obesity/adult/causes.html. 
2. Nelson MC, Story M, Larson NI, Neumark-Sztainer D, Lytle LA. Emerging adulthood and college-aged youth: an overlooked age for weight-related behavior change. Obesity (Silver Spring). 2008;16:2205-11.

3. Gropper SS, Simmons KP, Connell LJ and Ulrich PV. Weight and body composition changes during the first three years of College. J Obesity. 2012; 2012:6. Article ID 634048. https://doi.org/10.1155/2012/634048.

4. Gropper SS, Newton A, Harrington P, Simmons KP, Connell LJ, Ulrich P. Body composition changes during the first two years of university. Prev Med (Baltim). 2011;52:20-2.

5. Lloyd-Richardson EE, Bailey S, Fava JL, Wing R. Tobacco etiology research network (TERN). A prospective study of weight gain during the college freshman and sophomore years. Prev Med (Baltim). 2009;48:256-61.

6. Racette SB, Deusinger SS, Strube MJ, Highstein GR, Deusinger RH. Weight changes, exercise, and dietary patterns during freshman and sophomore years of college. J Am Coll Heal. 2005;53:245-51.

7. Anderson D, Shapiro J, Lundgren J. The freshman year of college as a critical period for weight gain: an initial evaluation. Eat Behav. 2003;4:363-7.

8. Gordon-Larsen P, Adair LS, Nelson MC, Popkin BM. Five-year obesity incidence in the transition period between adolescence and adulthood: the National Longitudinal Study of adolescent health. Am J Clin Nutr. 2004;80: 569-75.

9. Reis JP, Loria CM, Lewis CE, Powell-Wiley TM, Wei GS, Carr JJ, et al. Association between duration of overall and abdominal obesity beginning in young adulthood and coronary artery calcification in middle age. JAMA 2013:310:280.

10. Campbell MK. Biological, environmental and social influences on childhood obesity. Pediatr Res. 2016;79:205-11.

11. Nicklas TA, Baranowski T, Cullen KW, Berenson G. Eating patterns, dietary quality and obesity. J Am Coll Nutr. 2001;20:599-608.

12. Hill JO, Wyatt HR. Role of physical activity in preventing and treating obesity. J Appl Physiol. 2005;99:765-70.

13. Davis CD. The gut microbiome and its role in obesity. Nutr Today. 2016;51: 167-74

14. Parekh PJ, Balart LA, Johnson DA. The influence of the gut microbiome on obesity, metabolic syndrome and gastrointestinal disease. Clin Transl Gastroenterol. 2015:6:e91.

15. U.S. Department of Health and Human Services, National Institutes of Health. Managing Overweight and Obesity in Adults: Systematic Evidence Review from the Obesity Expert Panel. 2013.

16. Turnbaugh PJ, Hamady M, Yatsunenko T, Cantarel BL, Duncan A, Ley RE, et al. LETTERS a core gut microbiome in obese and lean twins. Nature. 2009; 457:480-4.

17. Turnbaugh PJ, Ley RE, Mahowald MA, Magrini V, Mardis ER, Gordon JI. An obesity-associated gut microbiome with increased capacity for energy harvest. Nature. 2006:444:1027-31.

18. Caspersen CJ, Pereira M a, Curran KM. Changes in physical activity patterns in the United States, by sex and cross-sectional age. Med Sci Sports Exerc 2000;32:1601-9.

19. Gordon-Larsen P, Nelson MC, Popkin BM. Longitudinal physical activity and sedentary behavior trends: adolescence to adulthood. Am J Prev Med. 2004; 27:277-83

20. American College Health Association, National College Health Assessment. Undergraduate reference group executive summary. 2014.

21. Cluskey M, Grobe D. College weight gain and behavior transitions: male and female differences. J Am Diet Assoc. 2009;109:325-9.

22. Butler SM, Black DR, Blue CL, Gretebeck RJ. Change in diet, physical activity, and body weight in female college freshman. Am J Health Behav. 2004;28:24-32.

23. Levitsky DA, Halbmaier CA, Mrdjenovic G. The freshman weight gain: a model for the study of the epidemic of obesity. Int J Obes. 2004:28:1435-42.

24. Demory-Luce D, Morales M, Nicklas T, Baranowski T, Zakeri I, Berenson G. Changes in food group consumption patterns from childhood to young adulthood: the Bogalusa heart study. J Am Diet Assoc. 2004;104:1684-91.

25. García-Meseguer MJ, Delicado-Soria A, Serrano-Urrea R. Fiber patterns in young adults living in different environments (USA, Spain, and Tunisia). Anthropometric and Lifestyle Characteristics Nutrients. 2017;9:1030.

26. Turnbaugh PJ, Ridaura VK, Faith JJ, Rey FE, Knight R, Gordon J. The effect of diet on the human gut microbiome: a metagenomic analysis in humanized gnotobiotic mice. Sci Transl Med. 2009;1:6ra14.

27. David LA, Maurice CF, Carmody RN, Gootenberg DB, Button JE, Wolfe BE, et al. Diet rapidly and reproducibly alters the human gut microbiome. Nature. 2013;505.
28. De Filippo C, Cavalieri D, Di Paola M, Ramazzotti M, Poullet JB, Massart S, et al. Impact of diet in shaping gut microbiota revealed by a comparative study in children from Europe and rural Africa. Proc Natl Acad Sci U S A. 2010:107:14691-6.

29. Deshpande S, Basil MD, Basil DZ. Factors influencing healthy eating habits among college students: an application of the health belief model. Health Mark Q. 2009;26:145-64.

30. Mitchell CM, Davy BM, Hulver MW, Neilson AP, Bennett BJ, Davy KP. Does Exercise Alter Gut Microbial Composition?-A Systematic Review. Med Sci Sport Exerc. 2018:Epub ahead of print.

31. Bressa C, Bailén-Andrino M, Pérez-Santiago J, González-Soltero R, Pérez M, Montalvo-Lominchar MG, et al. Differences in gut microbiota profile between women with active lifestyle and sedentary women. PLoS One. 2017;12.

32. Stewart CJ, Nelson A, Campbell MD, Walker M, Stevenson EJ, Shaw JA, et al. Gut microbiota of type 1 diabetes patients with good glycaemic control and high physical fitness is similar to people without diabetes: an observational study. Diabet Med. 2017:34:127-34.

33. Allen JM, Mailing $L$, Niemiro GM, Moore R, Cook MD, White BA, et al. Exercise alters gut microbiota composition and function in lean and obese humans. Med Sci Sport Exerc. 2018:50:747-57.

34. Clarke SF, Murphy EF, O'Sullivan O, Lucey AJ, Humphreys M, Hogan A, et al. Exercise and associated dietary extremes impact on gut microbial diversity. Gut. 2014:1-8.

35. Paulsen JA, Ptacek TS, Carter SJ, Liu N, Kumar R, Hyndman L, et al. Gut microbiota composition associated with alterations in cardiorespiratory fitness and psychosocial outcomes among breast cancer survivors. Support Care Cancer. 2017;25:1563-70.

36. OToole PW, Claesson MJ. Gut microbiota: changes throughout the lifespan from infancy to elderly. Int Dairy J. 2010;20:281-91.

37. Yatsunenko T, Rey FE, Manary MJ, Trehan I, Dominguez-Bello MG, Contreras $M$, et al. Human gut microbiome viewed across age and geography. Nature. 2012:486:222-7.

38. Jung ME, Bray SR, Ginis KAM. Behavior change and the freshman 15: tracking physical activity and dietary patterns in 1st-year University women. J Am Coll Heal. 2008;56:523-30.

39. Institute of Medicine. Dietary reference intakes for energy, carbohydrate, fiber, fat, fatty acids, cholesterol, protein, and amino acids (2002/2005). 2005

40. Koliada A, Syzenko G, Moseiko V, Budovska L, Puchkov K, Perederiy V, et al. Association between body mass index and Firmicutes/Bacteroidetes ratio in an adult Ukrainian population. BMC Microbiol. 2017;17:120.

41. Mathur R, Barlow GM. Obesity and the microbiome. Expert Rev Gastroenterol Hepatol. 2015;9:1087-99.

42. Sweeney TE, Morton JM. The human gut microbiome: a review of the effect of obesity and surgically induced weight loss. JAMA Surg. 2013;148:563.

43. Zhang H, DiBaise JK, Zuccolo A, Kudrna D, Braidotti M, Yu Y, et al. Human gut microbiota in obesity and after gastric bypass. Proc Natl Acad Sci U S A. 2009:106:2365-70.

44. Duncan SH, Lobley GE, Holtrop G, Ince J, Johnstone AM, Louis P, et al. Human colonic microbiota associated with diet, obesity and weight loss. Int J Obes. 2008;32:1720-4.

45. Hu H-J, Park S-G, Jang HB, Choi M-G, Park K-H, Kang JH, et al. Obesity Alters the Microbial Community Profile in Korean Adolescents. PLoS One. 2015;10; e0134333.

46. Schwiertz A, Taras D, Schäfer K, Beijer S, Bos N a, Donus C, et al. Microbiota and SCFA in lean and overweight healthy subjects. Obesity (Silver Spring). 2010;18:190-5

47. Wu GD, Chen J, Hoffmann C, Bittinger K, Chen Y-Y, Keilbaugh SA, et al. Linking long-term dietary patterns with gut microbial enterotypes. Science. 2011;334:105-8.

48. Walker AW, Ince J, Duncan SH, Webster LM, Holtrop G, Ze X, et al. Dominant and diet-responsive groups of bacteria within the human colonic microbiota. ISME J. 2011:5:220-30.

49. Welly RJ, Liu TW, Zidon TM, Rowles JL, Park YM, Smith TN, et al. Comparison of diet versus exercise on metabolic function and gut microbiota in obese rats. Med Sci Sports Exerc. 2016;48:1688-98.

50. Evans CC, LePard KJ, Kwak JW, Stancukas MC, Laskowski S, Dougherty J, et al. Exercise prevents weight gain and alters the gut microbiota in a mouse model of high fat diet-induced obesity. PLoS One. 2014:9:e92193.

51. Choi JJ, Eum SY, Rampersaud E, Daunert S, Abreu MT, Toborek M. Exercise attenuates PCB-induced changes in the mouse gut microbiome. Environ Health Perspect. 2013;121:725-30. 
52. Denou E, Marcinko K, Surette MG, Steinberg GR, Schertzer JD. High intensity exercise training increases the diversity and metabolic capacity of the mouse distal gut microbiota during diet-induced obesity. Am J Physiol Endocrinol Metab. 2016.

53. Queipo-Ortuño MI, Seoane LM, Murri M, Pardo M, Gomez-Zumaquero JM, Cardona $\mathrm{F}$, et al. Gut microbiota composition in male rat models under different nutritional status and physical activity and its association with serum leptin and ghrelin levels. PLoS One. 2013;8:e65465.

54. Kang SS, Jeraldo PR, Kurti A, Miller ME, Cook MD, Whitlock K, et al. Diet and exercise orthogonally alter the gut microbiome and reveal independent associations with anxiety and cognition. Mol Neurodegener. 2014;9:36.

55. Lambert JE, Myslicki JP, Bomhof MR, Belke DD, Shearer J, Reimer RA. Exercise training modifies gut microbiota in normal and diabetic mice. Appl Physiol Nutr Metab. 2015;40:749-52.

56. Petriz BA, Castro AP, Almeida JA, Gomes CP, Fernandes GR, Kruger RH, et al. Exercise induction of gut microbiota modifications in obese, non-obese and hypertensive rats. BMC Genomics. 2014;15.

57. Simpson HL, Campbell BJ. Review article: dietary fibre-microbiota interactions. Aliment Pharmacol Ther. 2015;42:158-79.

58. Lattimer JM, Haub MD. Effects of dietary fiber and its components on metabolic health. Nutrients. 2010;2:1266-89.

59. Asp NG. Resistant starch--an update on its physiological effects. Adv Exp Med Biol. 1997:427:201-10.

60. Lin A, Bik EM, Costello EK, Dethlefsen L, Haque R, Relman DA, et al. Distinct distal gut microbiome diversity and composition in healthy children from Bangladesh and the United States. PLoS One. 2013;8:e53838.

61. Petersen LM, Bautista EJ, Nguyen H, Hanson BM, Chen L, Lek SH, et al. Community characteristics of the gut microbiomes of competitive cyclists. Microbiome. 2017:5:98.

62. Mika A, Van Treuren W, González A, Herrera JJ, Knight R, Fleshner M, et al. Exercise is more effective at altering gut microbial composition and producing stable changes in lean mass in juvenile versus adult male F344 rats. PLoS One. 2015;10:e0125889.

63. Bruening M, van Woerden I, Todd M, Brennhofer S, Laska MN, Dunton G. A Mobile ecological momentary Assessment tool (devilSPARC) for nutrition and physical activity behaviors in college students: a validation study. J Med Internet Res. 2016;18:e209.

64. NHLBI. Clinical Guidelines on the Identification, Evaluation, and Treatment of Overweight and Obesity in Adults. NIH Publ. 1998;:98-4083.

65. Godin G, Shephard RJ. A simple method to assess exercise behavior in the community. Can J Appl Sport Sci. 1985;10:141-6.

66. Utter J, Neumark-Sztainer D, Jeffery R, Story M. Couch potatoes or french fries: are sedentary behaviors associated with body mass index, physical activity, and dietary behaviors among adolescents? J Am Diet Assoc. 2003; 103:1298-305.

67. Ma Y, Olendzki BC, Pagoto SL, Hurley TG, Magner RP, Ockene IS, et al. Number of 24-hour diet recalls needed to estimate energy intake. Ann Epidemiol. 2009;19:553-9.

68. JOHNSON RK, DRISCOLL P, GORAN MI. Comparison of multiple-pass 24-hour recall estimates of energy intake with Total energy expenditure determined by the doubly labeled water method in young children. J Am Diet Assoc. 1996;96:1140-4.

69. ASA24 Automated Self-administered 24-hour Recall. 2014 https://asa24.nci.nih.gov/.

70. Blanton CA, Moshfegh AJ, Baer DJ, Kretsch MJ. The USDA automated multiple-pass method accurately estimates group Total energy and nutrient intake. J Nutr. 2006;136:2594-9.

71. Caporaso JG, Lauber CL, Walters WA, Berg-Lyons D, Lozupone CA, Turnbaugh PJ, et al. Global patterns of 16S rRNA diversity at a depth of millions of sequences per sample. Proc Natl Acad Sci U S A. 2011;108(Suppl 1):4516-22.

72. Caporaso JG, Lauber CL, Walters WA, Berg-Lyons D, Huntley J, Fierer N, et al. Ultra-high-throughput microbial community analysis on the Illumina HiSeq and MiSeq platforms. ISME J. 2012;6:1621-4.

73. Human Microbiome Project Consortium. A framework for human microbiome research. Nature. 2012;486:215-21.

74. Caporaso JG, Kuczynski J, Stombaugh J, Bittinger K, Bushman FD, Costello EK, et al. QIIME allows analysis of high-throughput community sequencing data. Nat Methods. 2010;7:335-6.
75. DeSantis TZ, Hugenholtz P, Larsen N, Rojas M, Brodie EL, Keller K, et al. Greengenes, a chimera-checked 16S rRNA gene database and workbench compatible with ARB. Appl Environ Microbiol. 2006;72:5069-72.

76. Lozupone C, Knight R. UniFrac: a new phylogenetic method for comparing microbial communities. Appl Environ Microbiol. 2005;71:8228-35.

77. Segata N, Izard J, Waldron L, Gevers D, Miropolsky L, Garrett WS, et al. Metagenomic biomarker discovery and explanation. Genome Biol. 2011;12:R60
Ready to submit your research? Choose BMC and benefit from:

- fast, convenient online submission

- thorough peer review by experienced researchers in your field

- rapid publication on acceptance

- support for research data, including large and complex data types

- gold Open Access which fosters wider collaboration and increased citations

- maximum visibility for your research: over $100 \mathrm{M}$ website views per year

At BMC, research is always in progress.

Learn more biomedcentral.com/submissions 